\title{
Influence des endiguements et remblais sur l'inondation de la plaine de l'Agly
}

\author{
The influence of dikes and embankments on the Agly plain floods
}

\author{
par A. Paquier \\ Cemagref
}

Building of dikes along downstream reach of Agly River has protected the low plain from the more frequent floods, which encourages the economic development. In order to estimate the hazard linked with flooding and to decide relatively to urbanism documents, an hydraulic modelling was performed using 2-D software RUBAR 20. The boundary of the flooded areas was drawn for the 100-years' flood. However, the main features of flooding may change due to the division of the plain by many embankments that could slow or deviate the flow. Moreover, the always possible breaching of the main dikes constitutes the more damaging hazard for some areas.

\section{I — ENDIGUEMENT ET INONDATION}

Les endiguements constituent une solution très fréquemment utilisée dans les problèmes d'inondation. Une fois déterminé un débit de projet, il est, en effet, relativement simple de déterminer le niveau associé à ce débit et d'en déduire le niveau du sommet de berges qui permet la protection. Selon les cas, en fonction des objectifs, le débit de projet correspondra au débit de pointe quinquennal, décennal, vingtennal, centennal,...

Les principales questions qu'on est conduit à se poser sont :

1) pendant les crues pour lesquelles la protection doit être efficace, quel est l'effet de l'endiguement sur les zones en aval ou en amont ?

2) du fait de la modification des lignes d'eau pendant ces mêmes crues (mais aussi pour les autres débits), quelles conséquences sont à attendre sur la morphologie du lit ?

3) pour les crues dont les débits dépassent le débit de projet, quelles seront les conséquences pour les zones qu'on souhaite protéger?

4) nul endiguement n'étant complètement sûr surtout sur plusieurs kilomètres, quelles seraient les conséquences d'une rupture sur les zones à protéger ?

La protection créée par l'endiguement a aussi un autre effet (souvent recherché) qui est de favoriser le développement économique ; du fait de l'augmentation de la période de retour des inondations, on assiste à une implantation d'activités à plus grande valeur ajoutée. Cela conduit inévitablement à des dommages plus importants pour une crue dépassant fortement la crue de projet.

La plaine de la Salanque le long du cours aval de l'Agly est utilisée ci-après pour illustrer certains de ces aspects. Le contexte général est celui de crues rapides du fait du climat méditerranéen et de la proximité des reliefs. Les délais d'annonce des crues et d'évacuation des personnes en sont donc réduits, ce qui peut entrainer, outre les inévitables dommages aux biens, des pertes en vies humaines.

\section{II — PRÉSENTATION DE L'ÉTUDE SUR L'AGLY}

\subsection{Contexte de l'étude}

Sur les vingt derniers kilomètres de son cours, l'Agly traverse une plaine d'altitude inférieure à $20 \mathrm{~m}$ NGF bordée au Nord par l'étang de Leucate. Avant la construction des endiguements, cette plaine était inondée fréquemment (en moyenne tous les 3 ans), ce qui gênait l'activité agricole et, plus généralement, la vie des quelques villages situés dans la plaine. En outre, pour des crues très importantes comme celle d'octobre 1940 (débit de pointe très supérieur à $2000 \mathrm{~m}^{3} / \mathrm{s}$ ), la quasi totalité de la plaine pouvait être inondée. L'endiguement (et le recalibrage de l'ensemble du lit mineur) sur 13 kilomètres achevé en 1974 avait pour but de laisser passer un débit de $1250 \mathrm{~m}^{3} / \mathrm{s}$ correspondant à une période de retour de l'ordre de 30 ans. Un déversoir fusible était prévu en amont des endiguements afin que, lors des fortes crues, les écoulements s'effectuent dans la plaine en rive gauche pour rejoindre l'étang de Leucate ; ainsi, la pérennité des digues était mieux assurée.

Depuis ces travaux, aucune crue très importante n'a eu lieu, ce qui a entraîné le sentiment d'une protection quasi totale. L'activité économique dans la plaine s'est donc développée : agriculture à forte valeur ajoutée (serres,...) mais aussi industrie et habitat en liaison avec le développement de 


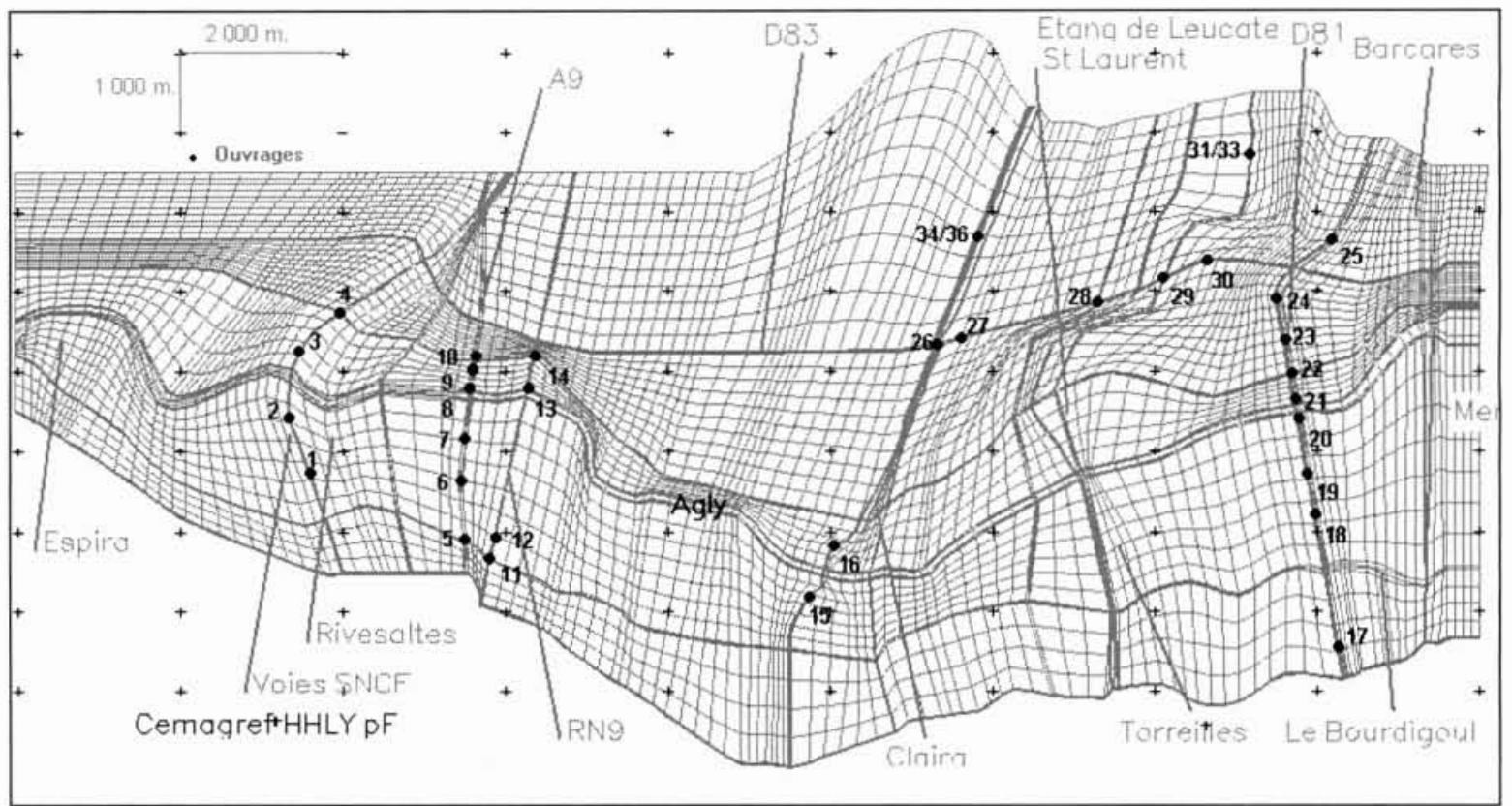

1. Zone d'étude.

la ville de Perpignan toute proche et avec l'essor touristique sur le littoral. Il était donc nécessaire de faire le point sur la protection réelle vis-à-vis des inondations afin d'éventuellement limiter cet accroissement très fort de la vulnérabilité de zones a priori toujours inondables. La construction d'un barrage sur le cours amont de l'Agly avec, en partie, un rôle d'écrêtement des crues, rendait indispensable cette étude puisque l'endiguement devenait efficace pour une période de retour plus élevée. La Direction Départementale de l'Equipement a donc chargé BRL associé au Cemagref de modéliser les inondations dans la plaine.

\subsection{Caractéristiques des simulations}

Pour simplifier, seule une crue centennale a été modélisée. Pour une crue beaucoup plus rare, on peut admettre que l'inondation tendrait vers la situation sans aménagement où la quasi totalité de la plaine était inondée. Il serait sans doute intéressant, au moins à titre pédagogique, de le vérifier. Pour une crue beaucoup plus fréquente, sauf accident, l'écoulement emprunterait uniquement le lit du fleuve.

La simulation d'une crue débordante requiert ici l'utilisation d'un modèle bidimensionnel. Un calcul unidimensionnel en réseau maillé exigerait la détermination (à partir de la topographie) de différents bras d'écoulement dans le lit majeur. En réalité, l'écoulement dans le lit du fleuve s'effectuerait en parallèle avec l'écoulement dans les zones les plus basses de la plaine, chenaux naturels souvent drainés par des fossés s'entrecoupant et se divisant plus ou moins selon l'ampleur de l'inondation. En outre, à ce cheminement relativement naturel, des obstacles assez nombreux s'opposent. Les principales voies de communication que sont la voie ferrée, l'autoroute A9, la RN9 et la RD81 (route littorale) sont, en partie, en remblai, tout particulièrement dans les zones basses, ce qui limite les écoulements de la crue d'Ouest en Est. De même, la RD83 qui est un axe Est-Ouest limite les écoulements SudNord vers l'étang de Leucate. Les ouvrages hydrauliques sous ces remblais (numérotés sur la figure 1) jouent alors un rôle majeur ; une estimation précise de leur capacité d'évacuation est donc indispensable. On notera cependant que, le jour de la crue, des atterrissements ou obstacles divers (arbres, voitures,...) peuvent réduire notablement cette capacité.

L'hydrogramme centennal injecté dans le modèle est un hydrogramme naturel type (débit de pointe de $2530 \mathrm{~m}^{3} / \mathrm{s}$ ) écrêté par le barrage sur l'Agly [1]. Deux formes d'hydrogramme (avec un ou deux pics) ont été testées sans différence notable dans les résultats dès l'instant que le volume au-dessus du seuil de débit de $1200 \mathrm{~m}^{3} / \mathrm{s}$ était similaire.

A l'aval, la mer et l'étang de Leucate ont été supposés supporter une surcote pendant la crue, les pluies importantes sur le bassin versant de l'Agly étant souvent accompagnées d'un fort vent d'Est susceptible d'entraîner des surélévations du niveau marin supérieures à un mètre.

L'ensemble des simulations a été effectué à l'aide du logiciel RUBAR 20 qui résout les équations de Saint Venant bidimensionnelles et permet de prendre en compte différents ouvrages hydrauliques. En outre, il simule des ruptures de digue instantanées ou progressives [2].

\section{III — RÉSULTATS DES SIMULATIONS}

L'hypothèse de base était le passage de la crue dans l'endiguement actuel, c'est-à-dire sans déversoir fusible et avec des cotes de sommet de digue plus ou moins régulières [3].

Le résultat est un écoulement de l'ordre de $1300 \mathrm{~m}^{3} / \mathrm{s}$ dans le lit principal aval sans déversement au-dessus des digues sauf localement en des points bas. Ceci est cohérent avec le débit du projet de recalibrage et inclut une légère diminution de la capacité du lit avec le temps. En l'absence d'érosion au droit de ces points bas des digues, les volumes déversés sont très faibles. L'endiguement a pour conséquence de relever le niveau par rapport à l'étalement dans la plaine aval. Il y a alors débordement juste à l'amont de la zone endiguée comme cela s'était passé lors de la crue de 1992 (débit de pointe estimé à $1210 \mathrm{~m}^{3} / \mathrm{s}$ ). 


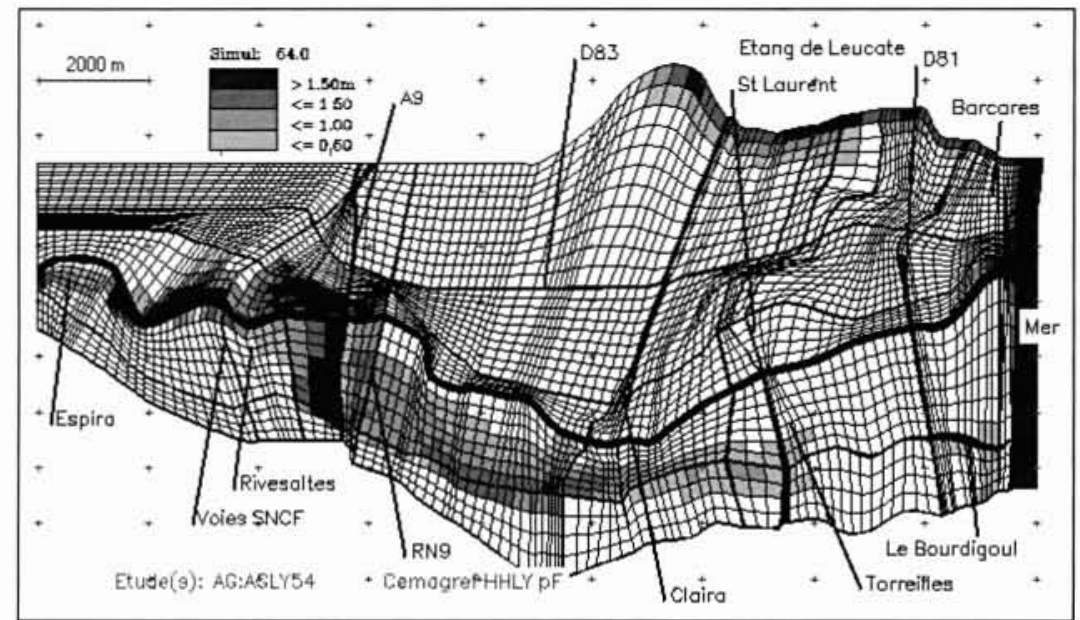

2. Etendue des zones submergées. voie ferrée, A9, RN9, chacun d'entre eux créant à son amont une zone inondée plus ou moins importante en fonction de la topographie, de la hauteur du remblai et des capacités d'évacuation et d'alimentation. La figure 2 donne un exemple d'étendue des zones submergées sachant que plusieurs variantes ont été testées. Aucun événement ne permettait, en effet, un calage précis des coefficients de frottement : la crue de 1992 n'a débordé que faiblement et son débit reste incertain (10 à $20 \%$ près ?) ; la crue de 1940 a un débit encore plus incertain et se situait dans un contexte d'occupation du sol bien différent.
En fait, pour une crue centennale, compte tenu des endiguements partiels, les débordements ont lieu sur plusieurs kilomètres et remontent jusqu'à la ville de Rivesaltes. Au total, c'est un volume de l'ordre de 10 millions de $\mathrm{m}^{3}$ qui se déverse en rive droite de l'Agly entre la ville de Rivesaltes et l'amont des endiguements. Du fait du remblai de l'autoroute, les volumes ne s'évacuent vers l'Est que lentement. Le retour vers le lit mineur de l'Agly est également limité par les endiguements, même si ceux-ci sont moins élevés qu'à l'aval. Cela entraîne un allongement de la durée de submersion audelà d'une journée alors que le déversement ne dure que 10 heures environ. Cela se traduit en revanche par une inondation d'ampleur plus limitée à l'aval de l'autoroute en rive droite d'autant plus que le ruisseau de la Llabanère permet d'évacuer un débit conséquent. De même, en rive gauche de l'Agly, les inondations sont limitées du fait de l'évacuation par les fossés et de la rétention à l'amont par les remblais :

\section{IV — CONSÉQUENCES D'UNE BRÈCHE}

Compte tenu d'un transit à plein bord pendant plusieurs heures dans le lit de l'Agly, la probabilité d'une rupture de l'endiguement est loin d'être négligeable. Selon la position et l'importance de la brèche, les conséquences sont évidemment différentes. Les simulations effectuées en supposant les ruptures instantanées au plus fort de la crue avec une largeur de brèche de 50 mètres ont montré que jusqu'à 10 millions de $\mathrm{m}^{3}$ pouvaient se déverser par une brèche avec un débit dépassant $200 \mathrm{~m}^{3} / \mathrm{s}$. Des ruptures moins pessimistes et sans doute plus réalistes (brèche moins large, rupture progressive plus tardive) pouvaient aussi être simulées. Elles n'auraient pas aussi bien permis de mettre l'accent sur le risque lié à la rupture de la digue, risque qui, en outre, augmente très fortement, en gravité et en probabilité d'occurrence, pour des crues plus rares.

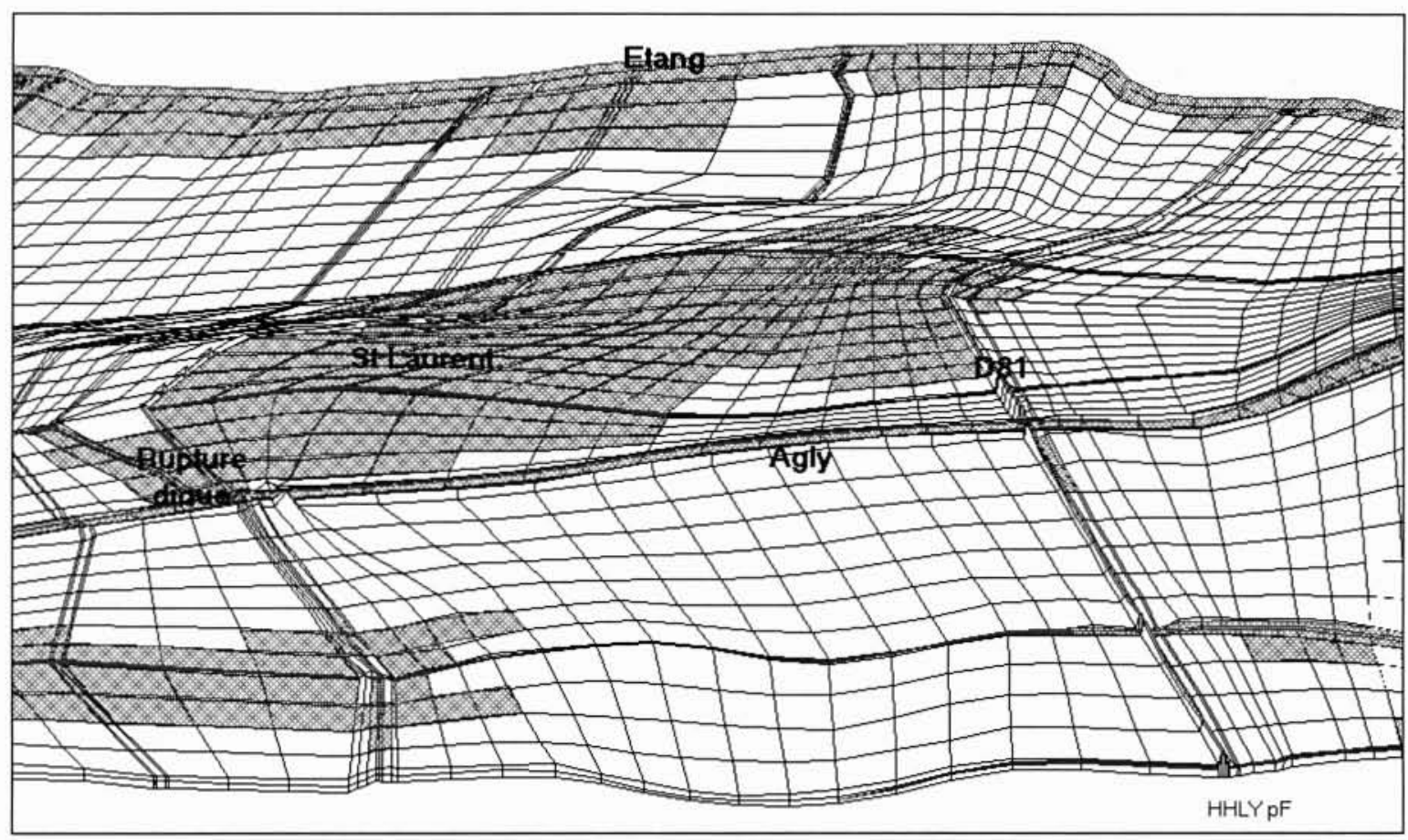

3. Inondation créée par la rupture d'une brèche. 
Pour de tels événements, les inondations peuvent être alors très importantes et très dangereuses pour les personnes. Toutefois, en général, les hauteurs de submersion restent faibles (inférieures au mètre), ce qui implique que les remblais routiers même de faible hauteur constituent un obstacle. Cela apparaît clairement sur la figure 3 où l'inondation est limitée à l'Est par la RD81, au Nord par la RD83 et à l'Ouest par la digue protégeant le village de Saint-Laurent des inondations en provenance de l'Ouest. L'évacuation des eaux se produit par les différents fossés et ouvrages sous les remblais ; c'est aussi par ces ouvrages ou par les endroits où les remblais sont de moindre hauteur que l'inondation s'étend légèrement au-delà des limites indiquées ci-dessus.

\section{$\mathrm{V} \square$ CONCLUSIONS}

Les faibles hauteurs de submersion dans le lit majeur impliquent que les remblais routiers (et les ouvrages permettant aux écoulements de les franchir) jouent un rôle important dans la propagation de l'inondation. Il est essentiel de bien les décrire : cotes de déversement, capacité d'évacuation des ouvrages. De même, le rôle exact joué par l'endiguement du lit mineur va dépendre de la capacité du lit et des cotes de sommet des digues. On rappelle que, lors d'un événement réel, des "incidents" peuvent modifier les écoulements : obstruction d'un ouvrage, rupture de remblai,... En particulier, l'aléa lié à la rupture d'un endiguement, bien que ne pouvant être aisément défini en termes de probabilité d'occurrence, est réel. En outre, sa probabilité augmente fortement dès qu'un déversement imprévu se produit d'où l'intérêt de prévoir des ouvrages (tels les seuils fusibles) qui permettent de limiter les sollicitations en orientant les déversements vers des zones moins vulnérables.
Selon les circonstances et la position du remblai ou de l'endiguement, on peut assister à une aggravation ou à une amélioration dans les zones non directement protégées qu'elles soient situées en amont ou en aval. La simulation des crues de différentes périodes de retour est souvent nécessaire pour conclure. Pour les zones protégées par des endiguements, la rupture constitue un aléa de faible probabilité mais pour lequel le danger pour les personnes est réel du fait de la rapidité de l'écoulement et de son caractère impromptu ; les zones proches des digues ou d'altitude relativement basse sont les plus exposées.

La simulation des inondations à l'aide d'un modèle bidimensionnel (plutôt qu'unidimensionnel) permet de mieux représenter l'étalement des eaux à l'amont d'un remblai ainsi que la combinaison d'un écoulement en nappe et par fossés. La définition d'un très grand nombre de "détails" : buses, talus de faible hauteur,... n'assure toutefois pas forcément une plus grande précision des résultats, ces "détails" étant souvent inefficaces en réalité. Un modèle moins détaillé permettra d'effectuer plus aisément le grand nombre de simulations nécessaire pour prendre en compte l'incertitude sur l'hydrologie, le calage du modèle, le fonctionnement réel des ouvrages en crue, la possibilité de rupture des digues, etc.

\section{BIBLIOGRAPHIE}

[1] Etude des zones inondables de l'Agly à l'aval de Rivesaltes, étude hydrologique. BRL Ingénierie, 1995.

[2] Paquier A., Farissier P., Assessment of risks of flooding by use of a 2-D model, Destructive water : Water-Caused Natural Disasters-Their Abatment and Control. IAHS, Anaheim, California, pp. 137-143, 1996.

[3] PAQUIER A., FARISSIER P., Etude des inondations centennales dans le bief aval de l'Agly, Cemagref, 1996. 